\title{
Stability criteria for power amplifiers under mismatch effects
}

\author{
Almudena Suárez, Franco Ramírez, Sergio Sancho \\ University of Cantabria, Santander, Spain
}

\begin{abstract}
Potential instability of power amplifiers (PAs) under mismatch effects is analyzed, with emphasis on the impact of the PA termination at the fundamental frequency. A largesignal version of the $\mu$ factor is derived, considering the lower and upper sideband of the fundamental frequency as virtual observation ports. This requires the application of the conversion matrix approach to each steady-state solution obtained with harmonic balance when varying the fundamental termination. To evaluate the impact of the fundamental output load, a contour technique is used to assemble loads giving the same $\mu$ value. A stabilization procedure is provided, based on the mapping of the mismatched load onto the stable region of the fundamental termination Smith chart. The analysis is extended to consider the baseband frequency, using three virtual observation ports.
\end{abstract}

Index Terms - Stability analysis, bifurcation, antenna mismatch.

\section{INTRODUCTION}

Power amplifiers (PAs) that are stable under ideal $50 \Omega$ terminations may become unstable under antenna mismatch conditions in realistic wireless environments where the antenna input impedance is affected by objects in its vicinity [1]. In many cases, the application imposes circuit stability for any possible load condition, including highly reflective loads [2]. These widely reported problems constitute a serious concern for PA manufacturers and several simulation procedures have been proposed for their prediction at the design stage. In [3], pole-zero identification was systematically applied to distinguish between stable and unstable points of the load Smith chart. This analysis disregards the frequency variation of the load impedance, which under mismatch conditions will exhibit unknown frequency dependence and take different values at the various harmonic components and sidebands. In [4] two methodologies, intended for harmonic balance (HB), were proposed to predict large-signal instabilities. One of them is based on the detection of secondary Hopf bifurcations by linearizing the circuit about the periodic large-signal regime with the conversion-matrix approach [5]. A second methodology [4], applicable in the case of two dominant sidebands, is based on the definition of a scattering-type matrix, looking into the circuit output and considering the two dominant sidebands as two virtual ports. This method has enabled an extension of the Rollet stability criteria to largesignal operation under output mismatch effects. However, the work [4] assumed a particular (matched) termination condition
$\Gamma_{o}=0$ at the fundamental frequency $f_{i n}$. The generalization to any termination implies some analysis difficulties, since any change of $\Gamma_{o}$ leads to a different steady-state solution, which must be calculated with $\mathrm{HB}$. This is not the case when changing the termination impedances at the sideband frequencies, which do not affect this solution. There is also a need for a judicious analysis procedure in order to interpret the multiple data and understand the stability behavior under changes in $\Gamma_{o}$. Here, a method is presented based on the calculation of contour plots of the $\mu$ factor represented on the $\Gamma_{o}$ Smith chart. This procedure has enabled the derivation of a stabilisation criterion based on the mapping of the mismatched load onto the stable region of the contour-plot representation.

\section{TERMINATION IMPEDANCE AT FUNDAMENTAL FREQUENCY}

In most PAs, the $50 \Omega$ output load will be preceded by a filter limiting the impact of mismatch effects to a few harmonics and sidebands. Initially the relevant terminations considered will be those corresponding to $f_{\text {in }}$ and the sidebands $f_{\text {in }}-f$ and $f_{\text {in }}+f$. The potential instability analysis in [4] is based on the calculation of a scattering-type matrix defined at the PA output terminals (plane $A$ in Fig. 1). The calculation requires an initial extraction of a $2 \times 2$ impendence matrix $Z$, obtained by linearizing the circuit about the large-signal periodic regime with the conversion-matrix approach [6]. Assuming a steady-state signal with harmonic components $k f_{i n}$, where $k$ goes from $-\mathrm{NH}$ to $\mathrm{NH}$, the introduction of a perturbation frequency $f$, will give rise, through mixing effects, to the sidebands $k f_{i n}+f$. For the potential stability analysis performed here, the relevant sideband impedance terminations will be those corresponding to: $-f_{\text {in }}+f$ and $f_{\text {in }}+f$.

The stability analysis carried out here relies on the use of ideal filters, so that $f_{i n}$ is terminated with $\Gamma_{o}$, the sidebands $\pm f_{\text {in }}+f$ are terminated in open circuit and $k f_{\text {in }}+f$, where $k \neq \pm 1$, are terminated in the default value $\Gamma=0$. First (second) circuit contains a small signal current source at the lower (upper) sideband frequency [4]. Matrix transformation provides:

$$
S\left(\Gamma_{o}, f\right)=\left[\begin{array}{ll}
S_{l l}\left(\Gamma_{o}, f\right) & S_{l u}\left(\Gamma_{o}, f\right) \\
S_{u l}\left(\Gamma_{o}, f\right) & S_{u u}\left(\Gamma_{o}, f\right)
\end{array}\right]
$$

where the subindexes $l$ and $u$ refer to lower and upper sidebands. Here, the potential instability will be analyzed using a large-signal equivalent of the $\mu$ factor [5]: 


$$
\mu_{L S}=\frac{1-\left|S_{l l}\right|^{2}}{\left|S_{u u}-\Delta_{L S} S_{l l}^{*}\right|+\left|S_{l u} S_{u l}\right|}
$$

Provided that the circuit is stable when the sidebands are terminated in open circuit and short circuit (proviso), the absolute stability condition will be $\mu_{L S}>1$. The proviso is easily verified with pole-zero identification. For a thorough analysis, $\mu_{L S}$ must be evaluated for all passive $\Gamma_{o}$ and all the perturbation frequencies comprised in the interval $\left(0, f_{\text {in }}\right)$. One must take into account that the large-signal periodic solution will change with $\Gamma_{o}$. Thus, the thorough evaluation of $\mu_{L S}$ will be done through a sequence of two-stage analyses: a HB analysis and a perturbation analysis based on the conversionmatrix approach. A double sweep is carried out in the magnitude and phase of $\Gamma_{o},\left(0\right.$ to 1 in magnitude and 0 to $360^{\circ}$ in phase), performing a $\mathrm{HB}$ analysis for each $\Gamma_{o}$, followed by a circuit linearization with the conversion-matrix approach to calculate (1) and (2). In this conversion-matrix analysis, the perturbation frequency $f$ is swept from 0 to $f_{i n}$, which provides the double-dependence factor $\mu_{L S}\left(f, \Gamma_{o}\right)$.

Representing the whole set of functions $\mu_{L S}\left(f, \Gamma_{o}\right)$ versus $f$ (or, equivalently $f_{\text {in }}-f$ ) one can identify the critical perturbation frequencies, providing $\mu_{L S}<1$. On the other hand, for each critical frequency, there will be a region of the $\Gamma_{o}$ Smith Chart, corresponding to potentially unstable terminations at the fundamental frequency. At each frequency, this will be delimited by the contour plot $\mu_{L S}\left(f_{c}, \Gamma_{o}\right)=1$, traced on the $\Gamma_{o}$ Smith Chart. Here, for illustration, the analysis will be applied for the perturbation frequency providing the minimum $\mu_{L S}$, denoted $f_{c}$. Instead of restricting the above analysis to the limit value $\mu_{L S}=1$, we will consider several values of $\mu_{L S}$, which will allow an investigation of the geometry of the $\mu_{L S}$ contour plots in the $\Gamma_{o}$ Smith chart. At the particular frequency $f_{c}$, the contour plot of all the $\Gamma_{o}$ values providing the same $\mu_{L S}$ will be defined as:

$$
C_{\mu o}\left(f_{c}\right)=\left\{\Gamma_{o} / \mu_{L S}\left(f_{c}, \Gamma_{o}\right)=\mu_{o}\right\}
$$

The technique has been applied to the amplifier in Fig. 1, which is unconditionally stable under output mismatch effects in small-signal regime. However, increasing the input power $P_{i n}$, it becomes potentially stable. Fig. 2(a) and Fig. 2(b) show the contour plots of $\mu_{L S}$ at $f_{i n}-f_{c}=783.5 \mathrm{MHz}$ for $P_{i n}=-$ $36 \mathrm{dBm}$ (stable) and $-2 \mathrm{dBm}$ (potentially unstable inside the region delimited by $\mu_{L S}=1$ ). The analysis demonstrates the continuity of the $\mu_{L S}$ contours.

Being a large-signal extension of the $\mu$ factor [5], $\mu_{L S}$ should have the same geometrical meaning: it should concord with the distance between the centre of the Smith chart at the upper sideband $\Gamma_{u}\left(\right.$ at $\left.f_{\text {in }}+f\right)$ and the edge of the stability circle. This stability circle will be boundary between $\Gamma_{u}$ loads giving negative and positive resistance when looking into the circuit output at $-f_{i n}+f_{p}$, thus corresponding to potential instability under mismatch effects. To verify this, the termination at $f_{\text {in }}$ will be fixed to the value $\Gamma_{\mathrm{o}}=0.5 \angle 270^{\circ}$, belonging to the unstable region in Fig. 2(b). With this fixed termination at $f_{i n}$, the coefficient $\mu_{L S}$ has been analysed versus the perturbation frequency $f$. Two particular $f$ values, indicated in Fig. 3(a) have been selected. For each of these two frequency values, the stability circle has been represented in the $\Gamma_{u}$ plane [Fig. 3(b)]. Comparison with Fig. 3(a) demonstrates that $\mu_{L S}$ does agree with the distance to the stability circle.

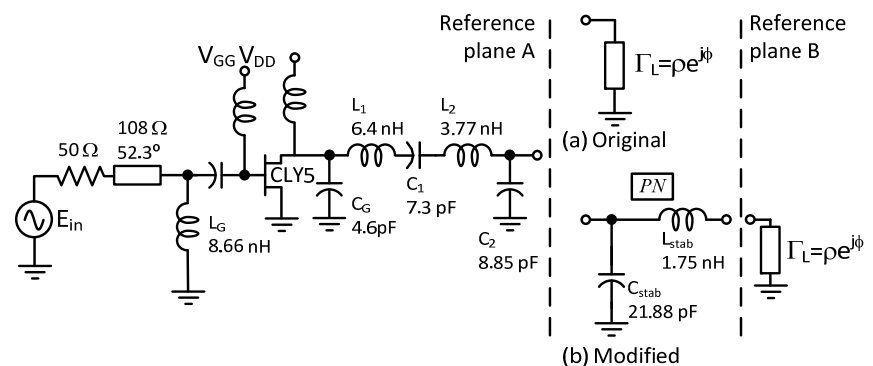

Fig. 1 Demonstrator PA at $f_{i n}=0.8 \mathrm{GHz}$. (a) Unstable, and (b) Stable.
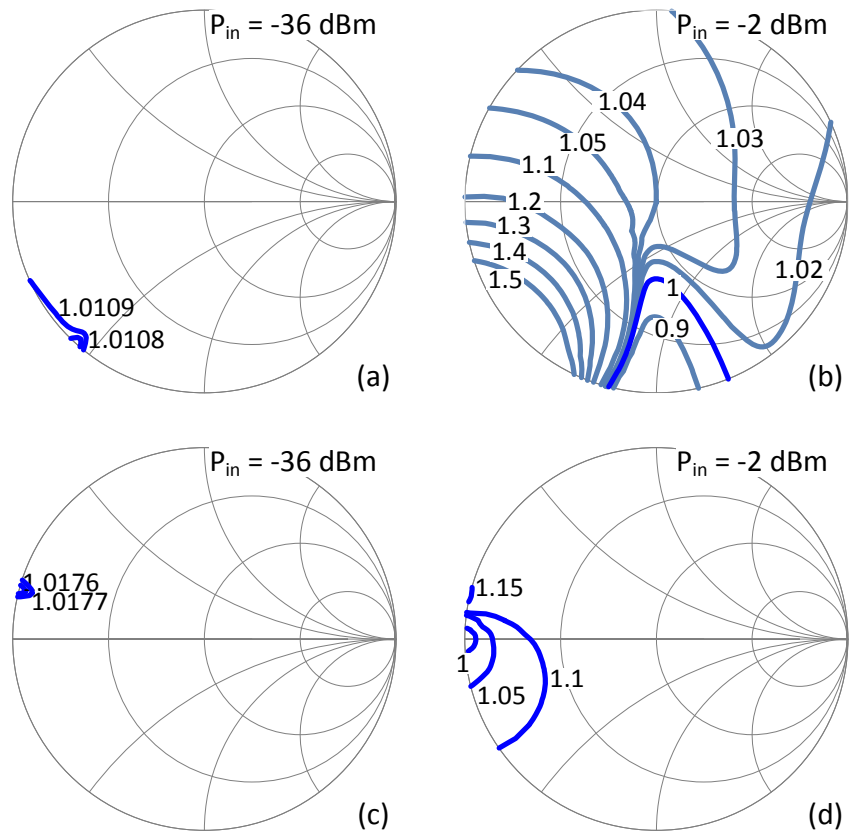

Fig. 2 Stability contours for two different $P_{\text {in }}$ values at the critical frequency 783.5 MHz. (a), (b) Original circuit. (c), (d) Modified circuit with very small unstable region (inside the $\mu_{L S}=1$ contour).

The fact that the stability circle in the $\Gamma_{u}$ plane constitutes a boundary between loads giving rise to negative and positive resistance at the lower frequency sideband $-f_{i n}+f$ is validated next. For this validation we will synthesize several loads, all with the same reflection coefficient $\Gamma_{\mathrm{o}}=0.5 \angle 270^{\circ}$ at $f_{\text {in }}$ and different values of $\Gamma_{u}$, inside and outside the larger stability circle in Fig. 3(b), corresponding to $f_{i n}-f_{p}=783.5 \mathrm{MHz}$. The tested $\Gamma_{u}$ loads are indicated with black dots in Fig. 3(b). Three loads have been synthesised with $R, L$ and $C$ elements, ensuring that each exhibits $\Gamma_{\mathrm{o}}=0.5 \angle 270^{\circ}$ at $f_{\text {in }}$ and the value of one of the black dots in Fig. 3(b) at $f_{i n}+f_{p}$. For the circle is valid boundary of potentially stability, synthesized loads with $\Gamma_{u}$ inside (outside) this stability circle should give rise to negative (positive) real part of the impedance seen when looking into the circuit at the lower sideband $-f_{i n}+f_{p}$. 


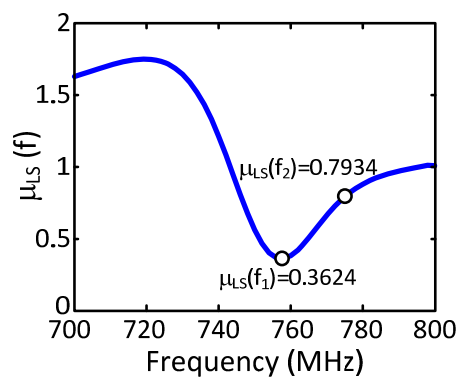

(a)

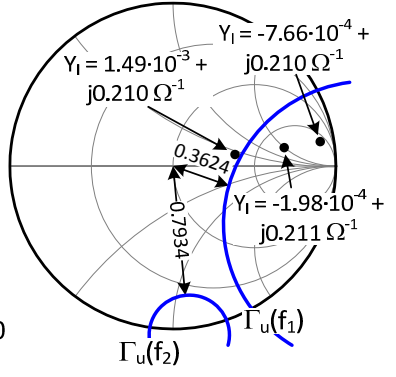

(b)
Fig. $3 \Gamma_{\mathrm{o}}=0.5 \angle 270^{\circ}$. (a) Variation of $\mu_{L S}$ vs the perturbation frequency $f$. (b) Stability circles for the two $\mu_{L S}$ in (a). The input admittance looking into the circuit at the lower sideband is indicated.

Each of the synthesized loads has been connected to the circuit output (plane $A$ in Fig. 1) and the input impedance when looking towards the circuit output from plane $A$ at $-f_{\text {in }}+f$ has been calculated with the conversion matrix approach. This analysis takes into account the actual impedance values exhibited by each synthesized load at all the sideband frequencies $k f_{\text {in }}+f$. The input impedance obtained at $-f_{\text {in }}+f_{p}$ for each black dot termination at $f_{i n}+f_{p}$ is indicated in the same figure [Fig. 3(b)]. Results confirm that the stability circle constitutes a boundary between the $\Gamma_{u}$ values giving negative and positive resistance at the lower sideband.

Additionally, the coefficient $\mu_{L S}{ }^{\prime}$, obtained by replacing $l$ with $u$ and vice versa, has also been calculated at the same perturbation frequency considered in Fig. 2. The $\mu_{L S}{ }^{\prime}$ contours, such that $\mu_{L S}{ }^{\prime}>1$, have been traced in Fig. 4. As expected, they are all located (red/dotted curves) in the unstable region already obtained with the analysis of Fig. 2(b), in terms of $\mu_{L S}$. The two contours $\mu_{L S}=1$ and $\mu_{L S}{ }^{\prime}=1$ are totally overlapped, since, in a manner similar to the small signal case [5], instability can be detected with any of these two coefficients. The coefficient $\mu_{L S}$ ' concords with the distance between the canter of the Smith chart associated with the upper sideband $\Gamma_{u}$, at $f_{\text {in }}+f$.

\section{STABILIZATION TEST}

A test for the usefulness of the $\mu_{\mathrm{LS}}$ contour plots on the $\Gamma_{o}$ plane to stabilize the circuit against mismatch effects will also be carried out. A passive subnetwork $(P N)$ will be introduced between the original circuit output and the final $50 \mathrm{Ohm}$ load (where the antenna should be connected), that is, between the reference planes indicated as $A$ and $B$ in Fig. 1. At a given perturbation frequency, the circuit will be unconditionally stable if the passive subnetwork $P N$ maps all possible passive loads (the full Smith Chart) onto the stable region of $\Gamma_{o}$. In the case analysed in Fig. 2b, the unstable region comprises reactive impedances, so the network should necessarily include resistive components to stabilize the circuit for all the possible passive terminations. To avoid degradation in the PA performance, an L-C section is used here. The L-C section will map the inside of any circle $\rho e^{j \theta}$ (where $\theta$ goes from $0^{\circ}$ to $360^{\circ}$ ) of the Smith Chart at plane $B$ onto a certain region of the Smith Chart, when looking from the plane $A$ towards the circuit output, as indicated in Fig. 1(b). The mapping action will be indicated as $\operatorname{Map}_{\mathrm{PN}}\left(\rho e^{j \theta}\right)$ The maximum value of $\rho$, such that the mapped region belongs to the stable region of the $\Gamma_{o}$ plane in Fig. 2(b,) is $\rho_{o}=0.93$, for which tangency occurs (Fig. 4). Note that the $\mu_{L S}$ contours traced in $\Gamma_{o}$ plane (reference plane $A$ ) inherently take into account the fundamental frequency $f_{\text {in }}$ and the two sidebands $-f_{i n}+f$ and $f_{\text {in }}+f$. The effect of the stabilization network is shown by obtaining $\mu_{L S}$ contour plots of the modified PA (including the subnetwork $P N$ ) that are shown in Fig. 2(d). These contour plots have been traced considering the reference plane $B$ in Fig. 1, which is the actual termination plane of the modified circuit. In agreement with the map of Fig. 4, the circuit is not unconditionally stable at the considered perturbation frequency. However, the unstable zone is reduced to a very small region near the short-circuit termination.

In the case of a low-pass filter instead of a band-pass filter at the circuit output, there will be three sideband terminations with possible impact on the PA stability properties: the baseband termination $\Gamma_{b}=\Gamma(f)$, the lower sideband termination $\Gamma_{l}=\Gamma\left(-f_{i n}+f\right)$ and the upper sideband $\Gamma_{u}=\Gamma\left(f_{i n}+f\right)$. The previous formulation can be extended to analyse the impact of these three sidebands. This will be based on the calculation of a $3 \times 3$ scattering matrix through the simultaneous conversion-matrix analysis of three circuits terminated in $\Gamma_{\mathrm{o}}$ at $f_{\text {in }}$, in open circuit at $f,-f_{\text {in }}+f$ and $f_{\text {in }}+f$ and in $\Gamma=0$ at the rest of frequency components. Each subcircuit will contain an independent current source at one of the sidebands $I(f), I\left(-f_{\text {in }}+f\right), I\left(f_{\text {in }}+f\right)$. Then, the parameters of a $3 \times 3$ impedance matrix will be calculated as:

$$
\begin{aligned}
& Z_{b b}=\frac{V(f)}{I(f)}, Z_{l b}=\frac{V\left(-f_{i n}+f\right)}{I(f)}, Z_{u b}=\frac{V\left(f_{i n}+f\right)}{I(f)} \\
& Z_{b l}=\frac{V(f)}{I\left(-f_{i n}+f\right)}, Z_{l l}=\frac{V\left(-f_{i n}+f\right)}{I\left(-f_{i n}+f\right)}, Z_{u l}=\frac{V\left(f_{i n}+f\right)}{I\left(-f_{i n}+f\right)} \\
& Z_{b u}=\frac{V(f)}{I\left(f_{i n}+f\right)}, Z_{l u}=\frac{V\left(-f_{i n}+f\right)}{I\left(f_{i n}+f\right)}, Z_{u u}=\frac{V\left(f_{i n}+f\right)}{I\left(f_{i n}+f\right)}
\end{aligned}
$$

The $(3 \times 3)$ impedance matrix can be transformed into a $(3 \times 3)$ matrix of Scattering type $S_{3}$. Assuming a particular termination $\Gamma_{s b}$ at any of the three sidebands $\left(\Gamma_{b}, \Gamma_{l}\right.$, or $\left.\Gamma_{u}\right)$, it will be possible to reduce the $3 \times 3$ matrix $S_{3}$ to a $2 \times 2$ matrix $S_{2}$ [7], depending on $\Gamma_{s b}$, which can be either $\Gamma_{b}, \Gamma_{l}$, or $\Gamma_{u}$. In this way, three different $2 \times 2$ matrixes can be defined: $S_{2}\left(\Gamma_{b}\right)$, $S_{2}\left(\Gamma_{l}\right)$ and $S_{2}\left(\Gamma_{u}\right)$. In each case, the $2 \times 2$ matrix is calculated at the two sidebands that do not appear inside the parenthesis. This reduction to a $2 \times 2$ matrix of scattering type will enable the calculation of 3 different $\mu$ factors. Because $\mathrm{S}_{2}$ depends on $\Gamma_{s b}$, the $\mu$ factor will also depend on $\Gamma_{s b}$. Performing a linearization of the type (4) for each termination $\Gamma_{\mathrm{o}}$ at $f_{i n}$, we will have a double dependence on $\Gamma_{o}$ and $\Gamma_{s b}$ at each perturbation frequency. This can be expressed as: 


$$
\mu_{L S}=\mu_{L S}\left\{S_{2}\left[S_{3}\left(\Gamma_{\mathrm{o}}\right), \Gamma_{s b}\right]\right\}=\mu_{L S}\left(\Gamma_{\mathrm{o}}, \Gamma_{s b}\right)
$$

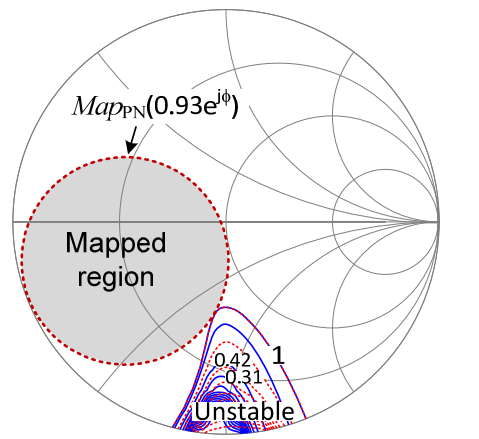

Fig. 4 Stabilization method by mapping the mismatched load onto the stable region of the $\Gamma_{o}$ chart, containing the $\mu_{L S}$ contour plots.

In this way, we will obtain three different functions: $\mu_{L S}\left(\Gamma_{o}, \Gamma_{u}\right), \mu_{L S}\left(\Gamma_{o}, \Gamma_{l}\right), \mu_{L S}\left(\Gamma_{o}, \Gamma_{b}\right)$. This analysis has been applied to the modified PA, calculating the three functions $\mu_{L S}\left(\Gamma_{0}, \Gamma_{u}\right), \mu_{L S}\left(\Gamma_{0}, \Gamma_{l}\right), \mu_{L S}\left(\Gamma_{0}, \Gamma_{b}\right)$ versus $f_{i n}-f($ Fig. 5). The calculation has been made for two different terminations at the fundamental frequency: $\Gamma_{o}=0.5 \angle-90^{\circ}$ (stable after the design modification) and $\Gamma_{o}=0.95 \angle 180^{\circ}$ (in the small potentially unstable region).
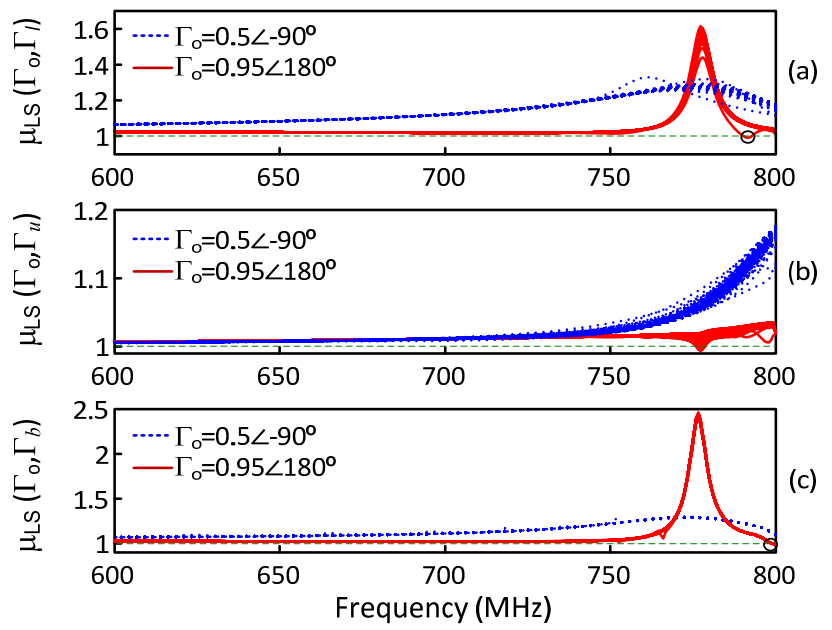

Fig. 5 Three-port stability analysis in terms for two $\Gamma_{o}$ values.

For each function $\mu_{L S}\left(\Gamma_{\mathrm{o}}, \Gamma_{u}\right), \mu_{L S}\left(\Gamma_{\mathrm{o}}, \Gamma_{l}\right), \mu_{L S}\left(\Gamma_{\mathrm{o}}, \Gamma_{b}\right)$, a double sweep has been performed in the sideband reflection coefficient (respectively corresponding to $\Gamma_{u}, \Gamma_{l}$ and $\Gamma_{b}$ ) have been considered, taking six equally spaced magnitudes, from 0.2 to 0.95 , phase values between $0^{\circ}$ and $360^{\circ}$, with a step of $15^{\circ}$. For $\Gamma_{o}=0.5 \angle-90^{\circ}$, the three functions fulfil $\mu_{L S}>1$ for all $f$. For $\Gamma_{o}=0.95 \angle 180^{\circ}$, the three functions fulfil $\mu_{L S}<1$ in a small frequency interval, in agreement with the small instability region predicted by Fig. 2(d) and Fig. 4.

The two PA versions have been connected to a wideband $\log$-periodic antenna. During the experiment, the antenna is connected to the amplifier through a low-loss feeder. This is sufficiently long to allow the positioning of the antenna in the vicinity of objects that give rise to undesired mismatch effects. The two amplifiers are stable in small signal. From $P_{i n}=-4 \mathrm{dBm}$, the original PA becomes unstable when the antenna is close to a reflective surface. Fig. 6 presents the measurements for $P_{i n}=-2 \mathrm{dBm}$. The original PA is unstable, with self-oscillating mixing behavior. The modified PA is stable for all the tested positions, which is attributed to the high $\left|\Gamma_{o}\right|$ in the small potentially unstable region.

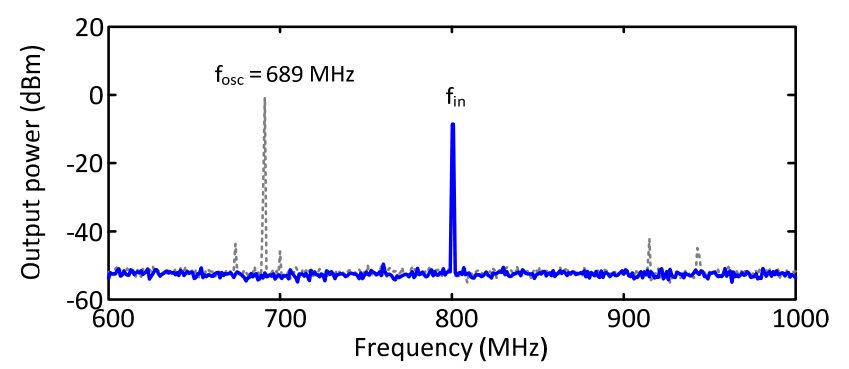

Fig. 6 Measurements of the original (gray dotted line) and modified (blue line) PA when connected to an antenna with $P_{i n}=-2 \mathrm{dBm}$.

\section{CONCLUSION}

The impact of the fundamental frequency termination on power amplifier instability under mismatch effects has been analyzed with a contour technique that enables the identification of stable and potentially unstable load values. Using these contours, a mapping method has also been derived for the amplifier stabilization. The methodology has been extended to the consideration of three relevant sidebands of interest in the case of circuits terminated with low-pass filters.

\section{ACKNOWLEDGEMENT}

Spanish project TEC2011-29264-C03-01 for financial support.

\section{REFERENCES}

[1] K. Narendra, E. Limiti, C. Paoloni, J. M. Collantes, R. Jansen, S. Yarman, "Vectorially Combined Distributed Power Amplifiers for Software-Defined Radio Applications," IEEE Trans. on Microw. Theory and Techn., vol. 60, no. 10, Oct., 2012.

[2] J.F. Imbornone, M. Murphy, R.S. Donahue, E. Heaney, "New insight into subharmonic oscillation mode of GaAs power amplifiers Under Severe Output Mismatch Condition," IEEE Journal of Solid State Circuits, vol. 32, pp. 1319-1325, Sept., 1997.

[3] A. Anakabe, et al. "Automatic pole-zero identification for multivariable large-signal stability analysis of RF and microwave circuits," Europ. Microw. Conf. (EuMC), Paris, pp. 477-480, 2010.

[4] A. Suárez, F. Ramírez, S. Sancho, "Stability analysis of power amplifiers under output mismatch effects," IEEE Trans. Microw. Theory Techn., vol. 62, no. 10, pp. 2273-2289, Oct., 2014.

[5] M. L. Edwards, J. H. Sinsky, "A new criterion for linear 2-port stability using geometrically derived parameters," IEEE Trans. Microw. Theory Techn., vol. 40, no. 12, pp. 2303-2311, Dec., 1992.

[6] P. Penfield, "Circuit theory of periodically driven nonlinear systems", Proceedings of the IEEE, vol. 54, No.2, pp. 266-280, Feb. 1966.

[7] E.L. Tan, "Simplified graphical analysis of linear three-port stability," IEE Proc. Microw. Antennas Propag., 152, 2005, pp. 209-213. 